\title{
Discriminação Simples com Mudanças Sucessivas na Função dos Estímulos: Aprendizagem em Bebês ${ }^{1}$
}

\author{
Flávia Teresa Neves Silva \\ Carlos Barbosa Alves de Souza ${ }^{2}$ \\ Universidade Federal do Pará
}

\begin{abstract}
RESUMO - Investigou-se em três bebês a aprendizagem de discriminações simples com mudanças sucessivas na função dos estímulos (DSMS). Figuras animadas foram apresentadas em uma tela sensível ao toque. O treino começou com uma discriminação simples (DS) com dois estímulos, com mudança na função dos estímulos, até que todos tivessem funcionado uma vez como S+ e outra como S-. Posteriormente, três estímulos foram apresentados na mesma tentativa, completando um ciclo de DSMS. Duas crianças aprenderam a DSMS com dois estímulos e uma, a DS com dois estímulos. A substituição dos conjuntos de estímulos durante o treino, caracterizada como um treino de múltiplos exemplares, parece ter favorecido a permanência das crianças na tarefa e a aprendizagem da mesma.
\end{abstract}

Palavras-chave: repertórios simbólicos; discriminação simples; discriminação condicional; bebês.

\section{Repeated Shifts of Simple Discrimination: Learning in Infants}

\begin{abstract}
This study investigated the acquisition of repeated shifts of simple discriminations (RSSD) in three infants. Animated images were presented on a touch sensitive screen. The procedure started off with a two-choice simple discrimination training (SD) with shifts in the stimulus function until all stimuli had functioned once as S+ and once as S-. Afterwards, all the three stimuli were presented in the same trial completing a RSSD cycle. Two of the infants learned the RSSD with the two-choice procedure and one infant learned the SD with two stimuli. The replacement of the stimulus sets throughout the training, characterized as a multiple exemplar training, seems to have contributed to the infants' engagement in the task as well as to the acquisition of the discriminations.
\end{abstract}

Keywords: symbolic repertoire; simple discrimination; conditional discrimination; infants.

O desenvolvimento da linguagem no Homo Sapiens parece estar baseado em alguns repertórios pré-requisitos (e.g., olhar-conjunto [mutual gazing], percepção categorial) presentes também em outras espécies (Hauser, Chomsky \& Fitch, 2002; Tomasello \& Call, 1997). A aquisição de repertórios relacionais arbitrários (e.g., discriminações condicionais, aprendizagem por exclusão) parece estar entre esses precursores da linguagem (Wilkinson \& Green, 1998). A identificação das condições necessárias e suficientes para o estabelecimento desses pré-requisitos será um passo importante para uma teoria evolutiva da cognição em geral e de seus componentes linguísticos específicos (Bates \& Elman, 2000). Um aspecto que parece ser importante e facilitador da aquisição de repertórios precursores da linguagem é a exposição precoce às condições relevantes (Deacon, 1997; Savage-Rumbaugh \& cols., 1993).

Nosso conhecimento atual dos repertórios visuais, auditivos e motores em crianças com desenvolvimento normal indica que esses repertórios apresentam um intenso desen-

1 Este trabalho é parte da Dissertação de Mestrado do primeiro autor, sob orientação do segundo autor, apresentada ao Programa de PósGraduação em Teoria e Pesquisa do Comportamento, da Universidade Federal do Pará. O trabalho foi financiado pelo Conselho Nacional de Desenvolvimento Científico e Tecnológico (CNPq) e pela Financiadora de Estudos e Projetos (FINEP).

2 Endereço para correspondência: Rua dos Mundurucus 822 Ap. 602. Jurunas. Belém, PA. CEP 66025-660.E-mail: carlos.souza@pesquisador.cnpq.br. volvimento no primeiro ano de vida (ver Bremner \& Fogel, 2004). No entanto, apesar do amplo conhecimento sobre os repertórios perceptuais-motores e de aprendizagem de relações contingenciais estímulo-estímulo e respostas-consequências (Rovee-Collie \& Barr, 2004), poucos estudos têm analisado o desenvolvimento de repertórios simbólicos e de seus pré-requisitos em suas etapas iniciais, i.e., em indivíduos com menos de 2 anos de vida. E muito menos ainda a partir de uma perspectiva analítico comportamental (cf. Horne \& Lowe, 1996; Zentall, Galizio \& Critchfield, 2002).

\section{Relações Condicionais, Equivalência e Linguagem em Crianças}

A ‘teoria da equivalência de estímulos’ tem sido proposta como uma forma de abordar a emergência de repertórios relacionais e simbólicos sob um ponto de vista analíticocomportamental (e.g., Sidman \& Tailby, 1982; Sidman, Wynne, Maguire \& Barnes, 1989). Dada uma história de aprendizagem, via treino direto, de discriminações condicionais arbitrárias entre estímulos, relações não diretamente treinadas podem ser aprendidas em função das propriedades intrínsecas às contingências de estabelecimento das relações condicionais: reflexividade, simetria e transitividade (Sidman, 2000).

A formação de classes de equivalência tem sido amplamente documentada em indivíduos com repertório verbal; por outro lado, a ocorrência desse fenômeno em indivíduos 
com limitado repertório verbal tem sido foco de controvérsias (Carr, Wilkinson, Blackman \& McIlvane, 2000; O’Donnel \& Saunders, 2003). Isso tem levado os pesquisadores da área a conduzir estudos com crianças pequenas, procurando avaliar a relação entre repertórios verbais e a formação de classes de equivalência.

Devany, Hayes e Nelson (1986) averiguaram se havia diferenças entre o desempenho de crianças com desenvolvimento normal e outras com déficit de linguagem em testes de equivalência. O estudo reuniu, em três grupos, 12 crianças com “idade mental” de 14 a 36 meses. Algumas crianças tinham desenvolvimento normal e outras não (estas distribuídas em subgrupos com e sem habilidade funcional de linguagem). Os autores informaram que todas as crianças com desenvolvimento normal tinham habilidades de fala condizentes com suas idades cronológicas, e que aquelas com déficit de linguagem foram assim identificadas com base no relato de seus professores e nas observações feitas pelos próprios experimentadores durante as sessões de treino e teste. As crianças foram expostas a um procedimento de discriminação condicional "gradual" de quatro conjuntos de dois estímulos visuais (as quatro relações foram ensinadas primeiro individualmente, depois aos pares e finalmente todas juntas em uma mesma sessão). Após ser alcançado o critério de aprendizagem na discriminação condicional com os quatros conjuntos apresentados na mesma sessão, foram realizados os testes para determinar se as classes de estímulos equivalentes eram formadas. Os resultados revelaram que todas as crianças com repertório verbal formaram classes de equivalência, ao passo que aquelas sem repertório verbal não o fizeram. Esses dados sugeriram uma relação entre o nível do repertório verbal das crianças e o desempenho em testes de equivalência.

Augustson e Dougher (1991) procuraram replicar os resultados de Devany e cols. (1986) realizando o mesmo tipo de treino "gradual”, mas utilizaram um computador para apresentar os estímulos e três estímulos de comparação a cada tentativa (Devany e cols. usaram dois). No primeiro experimento, quatro crianças com desenvolvimento normal, com idades variando entre 2 anos e 3 meses e 2 anos e 9 meses, não aprenderam sequer a tarefa de discriminação condicional. No segundo experimento, uma criança de 2 anos e 9 meses também não aprendeu a tarefa (mesmo após 211 tentativas), enquanto que duas crianças mais velhas (4 anos e 7 meses e 6 anos e 4 meses) aprenderam a tarefa de discriminação condicional em menos de 50 tentativas. Augustson e Dougher sugeriram que alterações realizadas por eles no procedimento podem ter "aumentado a complexidade do treino para além das capacidades desse grupo etário [crianças de 2 anos de idade]” (p. 24). Por outro lado, eles não apontaram quais variáveis poderiam explicar o sucesso das crianças de 4 e 6 anos na tarefa.

Lipkens, Hayes e Hayes (1993), em um estudo longitudinal com uma criança de 16 meses no começo do estudo e 27 meses no término, com desenvolvimento normal, verificaram a aprendizagem de relações condicionais arbitrárias entre estímulos e emergência de relações simétricas e transitivas. No entanto, de forma diferente de Devany e cols. (1986), a emergência das relações de derivação de tipo simétrica e transitiva pareceu não depender de um repertório verbal bem desenvolvido. De acordo com Lipkens e cols., esses desempenhos deveriam ser vistos como ações histórica e contextualmente situadas, desenvolvidas ao longo do tempo pela exposição a múltiplos exemplares de diferentes relações (i.e., a formação dos “quadros relacionais"”).

Boelens, Broek e Klarenbosch (2000) trabalharam com 14 crianças de 26 meses a 34 meses no início do estudo, com desenvolvimento normal, a fim de verificar a aprendizagem de discriminações condicionais arbitrárias e a emergência de simetria dessas relações. Primeiro, os participantes foram expostos a um treino de discriminação condicional por identidade com dois conjuntos de dois estímulos. Apenas 9 participantes aprenderam a tarefa e foram expostos ao procedimento de modelagem dos estímulos modelos das relações de identidade aprendidas, até transformá-las em relações condicionais arbitrárias, seguindo a proposta de Zygmont, Lazar, Dube e McIlvane (1992). Sete (dos nove) participantes aprenderam todas as discriminações condicionais arbitrárias e mostraram a emergência das relações de simetria. Boelens e cols. (2000) indicam que essa aprendizagem ocorreu sem nenhuma nomeação explícita dos estímulos, sugerindo que esses repertórios podem ser adquiridos na ausência de respostas verbais relacionadas. No entanto, eles reconhecem que em função da idade dos participantes, não se pode eliminar algum efeito de repertórios verbais sobre a aprendizagem das tarefas.

Considerados de maneira geral, os resultados dos estudos aqui analisados ainda deixam em aberto a questão do papel de repertórios verbais na aquisição de repertórios relacionais (discriminações condicionais) e na emergência de classes de equivalência. Parte da dificuldade está no fato dos estudos terem sido realizados, geralmente, com crianças com mais de 2 anos de idade, as quais já apresentam um repertório verbal bem desenvolvido e uma longa história de exposição a relações condicionais entre estímulos (cf. Horne \& Lowe, 1996; Souza \& Pontes, 2007). Além disso, como apontaram alguns autores (e.g., Augustson \& Dougher, 1991; Boelens \& cols., 2000), ainda sabemos pouco sobre as variáveis relevantes para a aquisição de repertórios condicionais arbitrários.

As dificuldades em atingir as discriminações condicionais arbitrárias, observadas nos estudos analisados, mostram a necessidade de investigar a aquisição de repertórios básicos, como a discriminação simples e condicional por identidade, que podem funcionar como base para o desenvolvimento dos repertórios simbólicos mais complexos (identidade generalizada, discriminação arbitrária e equivalência de estímulos) (cf. Barros, Galvão \& McIlvane, 2003; Boelens \& cols., 2000).

Recentemente, Gil e colaboradores (Gil \& Oliveira, 2003; Gil, Oliveira, Sousa \& Faleiros, 2006; Oliveira \& Gil, 2008) deram início a uma linha de pesquisa que tem procurado investigar as condições de aprendizagem de discriminações simples e condicionais por identidade em crianças entre 12 e 24 meses. Além das variáveis relacionadas com a aprendizagem desses repertórios, têm sido investigadas também

3 A formação de quadros relacionais implica o estabelecimento do responder relacional arbitrariamente aplicável com as seguintes propriedades: (a) interdependência mútua; (b) interdependência combinatória; e (c) transformação de função de estímulo (cf. Hayes, Barnes-Holmes \& Roche, 2001). 
as variáveis que favorecem a permanência das crianças nas situações experimentais, uma das grandes dificuldades no desenvolvimento de estudos com crianças pequenas.

De maneira geral, esses estudos demonstraram que o desempenho das crianças foi mais adequado quando foram manipuladas as seguintes variáveis: (1) controle da diversidade dos estímulos disponíveis no ambiente experimental; (2) aproximação da criança ao aparato experimental, de modo a aumentar as respostas de observação para os estímulos da tarefa; (3) adoção de um intervalo entre tentativas de aproximadamente 15 segundos; (4) presença de um adulto familiar durante as sessões experimentais; (5) introdução de um período de brincadeira com o experimentador com os estímulos com função positiva (S+) após cada acerto, e de um período de brincadeira livre com o experimentador após o encerramento das tarefas; e (6) realização de um número pequeno de tentativas por sessão (entre 5 e 12) e adoção de critérios de aprendizagem mais flexíveis.

O presente estudo procurou analisar em bebês, entre os 10 e 20 meses de vida, a aprendizagem de discriminações simples com mudanças sucessivas na função dos estímulos (DSMS). Tem sido sugerido que esse repertório pode facilitar o ensino de discriminações condicionais por identidade (DCI) a humanos com habilidades linguísticas limitadas e a não-humanos (ver Rico, 2006; Sidman \& cols., 1989, Souza, Borges, Goulart, Barros \& Galvão, 2009), discriminações estas que podem funcionar como base para o desenvolvimento de repertórios simbólicos mais complexos (tais como identidade generalizada, discriminação arbitrária e equivalência de estímulos) (Barros \& cols., 2003, Boelens \& cols., 2000; Zygmont \& cols., 1992). Além disso, com base no acompanhamento do desempenho dos bebês, buscou-se, ainda, avaliar o efeito de algumas variáveis (e.g., redução de estímulos no ambiente, variação de estímulos reforçadores) na manutenção dos participantes no ambiente experimental e na aprendizagem da tarefa.

\section{Método}

\section{Participantes}

Participaram do estudo três crianças experimentalmente ingênuas, sem problemas de visão, audição ou de saúde, segundo a informação dos pais. Duas crianças (P12 e P14) eram do sexo masculino e uma terceira (P10), do sexo feminino (os números na identificação de cada participante indicam a sua idade em meses no início do experimento). Aos responsáveis pelas crianças foi solicitado que assinassem um termo de consentimento livre e esclarecido, autorizando-as a participar do estudo (aprovado pelo comitê de ética em pesquisa do Centro de Ciências da Saúde/Universidade Federal do Pará - protocolo de n 145/06).

\section{Ambiente e Equipamentos}

As sessões experimentais foram realizadas em uma sala $\left(36 \mathrm{~m}^{2}\right)$ do Laboratório de Estudos do Comportamento
Simbólico da Universidade Federal do Pará. A sala conta com iluminação artificial e direta, é climatizada e possui dois espelhos one-way. O equipamento utilizado foi um computador Intel Pentium IV com tela de 17 pol. sensível ao toque, alguns brinquedos (bolas, bonecas, carrinhos, balões, fantoches, mini-instrumentos musicais, dentre outros) para facilitar o processo de interação com a criança antes e durante as sessões e um mini-veículo para conduzir a criança pela sala experimental até a tela do computador. O PowerPoint 2007 foi usado para a apresentação dos estímulos e consequenciação automática das respostas dos participantes. Todas as sessões foram filmadas por uma câmera digital fixada em um tripé em um dos cantos da sala.

\section{Estímulos}

Foram utilizadas 12 figuras animadas (formato GIF) com cores, formas e movimentos diferentes (ver Figura 1), aspectos que poderiam aumentar a discriminabilidade das mesmas (ver Slater, 2004). As figuras apareciam em um fundo branco ou preto de cerca de $5 \times 5 \mathrm{~cm}$ na tela do computador. Como em cada sessão poderiam ser apresentadas até três figuras (ver Procedimento e Tabela 1), elas foram divididas em quatro grupos (A, B, C e D) de três figuras cada.

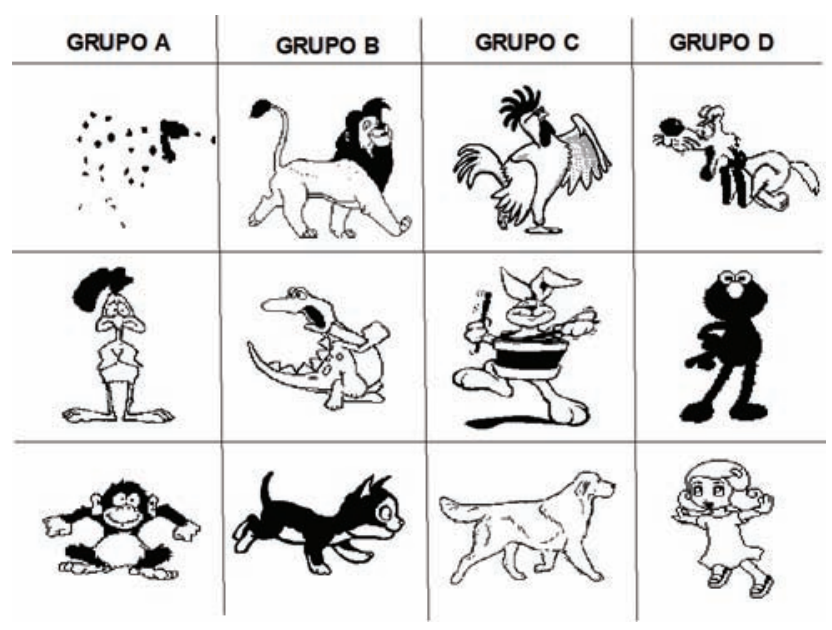

Figura 1. Estímulos experimentais.

\section{Procedimento}

No início do experimento foram realizadas algumas sessões de interação com a criança na sala experimental, por meio de jogos diversos com os brinquedos presentes na sala, procurando habituá-la ao novo ambiente e à presença da experimentadora (E). Após esse período de habituação teve início a modelagem e o treino de DSMS, com três a cinco sessões por semana (uma ou duas por dia, com duração máxima de 20 minutos cada). No início de cada sessão, a criança era conduzida por $\mathrm{E}$ até o local da sala onde se encontrava o computador. Durante as sessões foi solicitado ao responsável pela criança que permanecesse na sala experimental, sentado em um sofá, de modo a permanecer no campo visual do participante, mas sem interagir com ele nos momentos de apresentação dos estímulos. 
Em uma primeira sessão, foi verificado se a criança apresentava o comportamento de tocar algumas figuras (diferentes das usadas no experimento) apresentadas na tela do computador. Se essa resposta não ocorresse, ela era modelada: ou seja, eram realizadas apresentações de apenas um estímulo (GIF) na tela, e repostas de aproximação, espontânea ou guiadas pela E, à tela/estímulo eram reforçadas pela apresentação de filmes/desenhos na tela e interação com E. Após essa etapa teve início o treino de discriminação simples com mudanças sucessivas na função dos estímulos (DSMS).

Treino de DSMS. Foi adaptado um procedimento de treino de DSMS que se mostrou eficaz como treino preparatório para o estabelecimento de repertórios mais complexos, como a aprendizagem de discriminação condicional por identidade (DCI), em macacos-prego (Cebus apella) (Rico, 2006; Souza \& cols., 2009).

O procedimento foi adaptado para ser realizado com crianças, utilizando como estímulos figuras GIFs apresentadas na tela de um computador. Os estímulos eram apresentados em posições diferentes a cada tentativa (em uma matriz de ordem 3). No início do procedimento, uma tentativa correspondia à seguinte sequência de eventos: primeiro apenas um dos GIFs (Estímulo Isolado - EI) era apresentado na tela. Um toque da criança no GIF era consequenciado com o seu desaparecimento e a apresentação de um filme/desenho animado de 5 a $10 \mathrm{~s}$ de duração, ao final do qual eram apresentados dois (ou três) GIFs na tela. Um desses estímulos era idêntico ao EI e tinha a função de estimulo correto (S+). A escolha do $\mathrm{S}+$ era consequenciada com um filme/desenho animado de 5 a 10 s de duração e interações com E (palavras de elogio e brincadeiras sobre o filme/desenho). A escolha de um estímulo não idêntico (S-) era consequenciada com a apresentação de uma tela preta durante 5 a 10 s e não havia interações com E. Logo após o final do filme/desenho animado ou da tela preta tinha início uma nova tentativa.

Eram preparadas oito tentativas por sessão. As respostas dos participantes eram filmadas e posteriormente registradas pela E como: (1) Acertos - escolher S+; (2) Erros - escolher S-; e (3) Resposta com prompt - quando a criança olhava para os estímulos na tela sem tocá-los por cerca de $15 \mathrm{~s}$. Esse comportamento era consequenciado por $\mathrm{E}$ com prompts visuais (apontar para o $\mathrm{S}+$ ) e/ou físicos (levar a mão da criança ao $\mathrm{S}+$ ). Foram definidos três possíveis critérios de aprendizagem: (1) quatro respostas corretas consecutivas (rcc) na mesma sessão; (2) cinco rcc entre sessões (e.g., as duas últimas da sessão 1 e as três primeiras da sessão 2); ou (3) três rcc por sessão em três sessões consecutivas (desconsiderando as tentativas com prompts nos três casos).

Treino com dois estímulos. O treino foi planejado para iniciar com uma discriminação simples (DS) com dois estímulos (usando primeiro o Conjunto A) na mesma tentativa. Atingido um dos critérios de aprendizagem nessa primeira discriminação, procedia-se à mudança na função dos estímulos, sendo que o S- do treino anterior assumia a função de $\mathrm{S}+$, e um terceiro estímulo passava a funcionar como S- (para evitar um efeito de persistência comportamental; cf. Rico, 2006). Esse procedimento era mantido até que três estímulos diferentes funcionassem uma vez como S+ e outra como S-.
Treino com três estímulos. Em seguida, foi realizado um treino DS com três estímulos na mesma tentativa. As três primeiras tentativas apresentavam dois estímulos, um que tinha função de S- no treino imediatamente anterior, assumindo agora a função de $S+$, e outro que não estava presente no último arranjo treinado (para exercer a função de S-). Somente a partir da quarta tentativa é que era introduzido o segundo estímulo S- (o S+ da sessão anterior). Nessa etapa do treino discriminativo, considerou-se como critério de aprendizagem a apresentação de três rcc nas tentativas com três estímulos, na mesma sessão, ou de cinco rcc nas tentativas com três estímulos, entre sessões. O treino de DSMS com três estímulos deveria prosseguir até que cada um deles tivesse funcionado como S+ e S- (um ciclo de treino de DSMS) (ver Tabela 1).

Depois disso, o mesmo procedimento seria repetido com a redução de $50 \%$ do reforçamento nas apresentações do Estímulo Isolado (EI) a cada ciclo de treino, até a retirada total do reforço na apresentação do EI. Decréscimos no desempenho das crianças produziam um conjunto de alterações no procedimento (e.g. introdução de um novo conjunto de estímulos, redução dos estímulos no ambiente, variação nos estímulos reforçadores) que procuravam recuperar o desempenho e favorecer a permanência na situação experimental (como essas alterações no procedimento dependiam dos desempenhos dos participantes, elas são detalhadas na apresentação dos resultados).

\section{Resultados}

Em função do desempenho dos participantes, o treino de DSMS somente foi implementado até a primeira etapa com três estímulos e $100 \%$ de reforço nas apresentações do Estímulo Isolado, para P12 e P14, e até a etapa com dois estímulos, para P10. Os participantes P14 e P12 mostraram evidência da aprendizagem de DSMS com dois estímulos (ver tabelas 2 e 4, respectivamente) e P10, da aprendizagem de DS com dois estímulos (ver Tabela 3).

Como pode ser visto na Tabela 2, P14 passou pelo treino de oito arranjos de DS com dois estímulos, tendo alcançado

Tabela 1. Etapas do treino de DSMS.

\begin{tabular}{|c|c|c|c|c|c|}
\hline \multirow{2}{*}{$\begin{array}{c}\text { Estímulo } \\
\text { Isolado (EI) }\end{array}$} & \multicolumn{3}{|c|}{ Estímulos Simultâneos } & \multirow{2}{*}{$\begin{array}{c}\mathrm{N}^{\circ} \text { de } \\
\text { Tentativas }\end{array}$} & \multirow{2}{*}{$\begin{array}{c}\% \text { de } S^{R} \\
\text { no } E I^{1}\end{array}$} \\
\hline & $\mathrm{S}+$ & S- & S- & & \\
\hline $\mathrm{X} 1$ & $\mathrm{X} 1$ & $\mathrm{X} 2$ & & 8 & \multirow{9}{*}{$100 \%$} \\
\hline $\mathrm{X} 2$ & $\mathrm{X} 2$ & $\mathrm{X} 3$ & & 8 & \\
\hline $\mathrm{X} 3$ & $\mathrm{X} 3$ & $\mathrm{X} 1$ & & 8 & \\
\hline \multirow{2}{*}{$\mathrm{X} 1$} & $\mathrm{X} 1$ & $\mathrm{X} 2$ & - & 3 & \\
\hline & $\mathrm{X} 1$ & $\mathrm{X} 2$ & X3 & 5 & \\
\hline \multirow{2}{*}{$\mathrm{X} 2$} & $\mathrm{X} 2$ & X3 & - & 3 & \\
\hline & $\mathrm{X} 2$ & X3 & $\mathrm{X} 1$ & 5 & \\
\hline \multirow{2}{*}{ X3 } & $\mathrm{X} 3$ & $\mathrm{X} 1$ & - & 3 & \\
\hline & X3 & $\mathrm{X} 1$ & $\mathrm{X} 2$ & 5 & \\
\hline
\end{tabular}

${ }^{1}$ Porcentagem de reforço $\left(\mathrm{S}^{\mathrm{R}}\right)$ para resposta ao Estímulo Isolado (EI). 
Tabela 2. Número de tentativas e de sessões nas etapas do treino de DSMS de P14.

\begin{tabular}{cccccc}
\hline & Estímulos & & Tentativas & Sessões \\
\cline { 1 - 3 } S+ & S- & S- & & $3^{*}$ \\
A1 & A2 & - & 12 & 4 \\
B1 & B2 & - & 32 & 7 \\
B2 & B3 & - & 52 & 7 \\
B3 & B1 & - & 55 & $12^{*}$ \\
B1 & B2 & B3 & 89 & 4 \\
C3 & C1 & - & 31 & 4 \\
C1 & C2 & - & 32 & $5^{*}$ \\
C2 & C3 & C1 & 39 & 2 \\
D1 & D2 & - & 16 & $6^{*}$ \\
D2 & D3 & - & 46 & 54 \\
\hline
\end{tabular}

* Não foi alcançado nenhum dos critérios de aprendizagem

um dos critérios de aprendizagem em seis deles. Entre os arranjos de dois estímulos em que um dos critérios foi alcançado, três (B2/B3, B3/B1 e C1/C2) implicavam mudança na função de estímulo. Por outro lado, P14 passou duas vezes pelo treino de DS com três estímulos sem alcançar nenhum dos critérios de aprendizagem. Foram usados os quatro conjuntos de estímulos, com as substituições ocorrendo em função da dificuldade em alcançar um dos critérios de aprendizagem com determinado conjunto.

A Figura 2 apresenta as respostas por tentativas nas 54 sessões de P14. Pode-se ver que P14 apresentou dificuldade com o treino inicial com estímulos do conjunto A, não completando sequer as oito tentativas das três sessões realizadas com esse conjunto. Com a substituição dos estímulos do conjunto A por estímulos do conjunto B (sessão 4), as oito tentativas foram concluídas. A partir da $4^{\mathrm{a}}$ sessão, além da substituição do conjunto A pelo B, houve uma redução gradual dos brinquedos disponíveis no ambiente experimental, diminuindo os estímulos concorrentes com a tarefa. A troca dos conjuntos e a redução de estimulação no ambiente parecem ter sido efetivas para aumentar o engajamento do participante na tarefa e melhorar seu desempenho, como pode ser visto pelo desempenho de P14 nas sessões 5 a 21 .

Com a inclusão de um terceiro estímulo no treino de DSMS, P14 voltou a não concluir todas as tentativas de uma sessão, sendo usados prompts em algumas tentativas (ver sessões 22 a 28). A partir da sessão 30, as respostas incorretas de P14 passaram a ser consequenciadas pela experimentadora ("este não tem filme" ou "este não é o correto"). Também procurando garantir o engajamento de P14 na tarefa e melhorar seu desempenho, a partir da sessão 34 os vídeos (uma das consequências reforçadoras) passaram a ter sempre 10 segundos e não mais 5 ou 10 s. Além disso, eles passaram a ser apresentados em sequência, sendo que em cada sessão, cerca de três filmes diferentes eram apresentados em fragmentos (sendo trocados a cada duas ou três sessões).

Essas alterações, juntamente com a substituição dos conjuntos de estímulos (primeiro do conjunto B pelo C e depois desse pelo D), produziram uma melhora inicial no desempenho de P14, aumentando o número de sessões completadas (sem prompts) e o alcance de um critério de aprendizagem no treino de DSMS com dois estímulos com os conjuntos C e D (ver sessões 30 a 41 e 47 a 49). No entanto, essas alterações não foram suficientes para melhorar o desempenho de P14 no treino de DS com três estímulos. Além disso, verificou-se que, gradualmente, a interação verbal/social de correção das respostas erradas de P14 passou a ter função reforçadora (uma forma de interação com a experimentadora), produzindo um aumento no número de respostas aos S-. Por isso, a partir da sessão 50, foram retiradas as interações verbais e não-verbais para as respostas erradas de P14.

Conforme indicado na Tabela 3, P10 passou pelo treino de oito arranjos de DS com dois estímulos, tendo alcançado um dos critérios de aprendizagem em quatro. Somente um desses arranjos nos quais ela alcançou um critério de aprendizagem (A2/A3) implicava mudança na função de estímulo. Dessa forma, o desempenho de P10 não possibilitou que o treino de DSMS com dois estímulos fosse implementado e, consequentemente, os treinos com três estímulos também não ocorreram. No treino de DS foram usados os quatro conjuntos de estímulos, mas principalmente em função da adoção da estratégia de substituir o grupo de estímulos a cada cinco ou seis sessões sem critério de aprendizagem (a partir da sessão 25; ver Tabela 3 e Figura 3).

Na Figura 3 pode-se ver as respostas por tentativas nas 44 sessões realizadas com P10. Aqui também se verifica que a substituição dos conjuntos de estímulos produziu uma melhora no desempenho do participante em duas ocasiões: após a substituição dos estímulos do conjunto A pelos estímulos do $\mathrm{B}$, e do conjunto $\mathrm{C}$ pelos estímulos do conjunto $\mathrm{D}$, bastaram, respectivamente, duas sessões (26 e 27) e apenas uma (sessão 39) para que um dos critérios de aprendizagem fosse

Tabela 3. Número de tentativas e de sessões nas etapas do treino de DSMS de P10.

\begin{tabular}{|c|c|c|c|c|}
\hline \multicolumn{3}{|c|}{ Estímulos } & \multirow{2}{*}{ Tentativas } & \multirow{2}{*}{ Sessões } \\
\hline S+ & S- & $\mathrm{S}$ & & \\
\hline A1 & A2 & - & 79 & 12 \\
\hline A2 & A3 & - & 42 & 7 \\
\hline A3 & A1 & - & 47 & $6^{*}$ \\
\hline B3 & B1 & - & 15 & 2 \\
\hline B1 & B2 & - & 40 & $5^{*}$ \\
\hline C1 & $\mathrm{C} 2$ & - & 47 & $6^{*}$ \\
\hline D1 & D2 & - & 8 & 1 \\
\hline \multirow[t]{2}{*}{ D2 } & D3 & - & 38 & 5* \\
\hline & TOT & & 316 & 44 \\
\hline
\end{tabular}

* Não foi alcançado nenhum dos critérios de aprendizagem 




Figura 2. Respostas por tentativas nas 54 sessões de P14. O item acertos/tentativas corresponde aos acertos pelo número de tentativas completadas na sessão. As linhas horizontais contínuas indicam a mudança na função dos estímulos e as pontilhadas, mudança no grupo de estímulos treinados. O critério de aprendizagem de 4 rcc é indicado pelo número 1 sobrescrito no item acerto/ tentativa. ‘Estímulos Tocados’ caracterizam as respostas sem prompts e ‘Estímulos Indicados’, as respostas após prompts. 


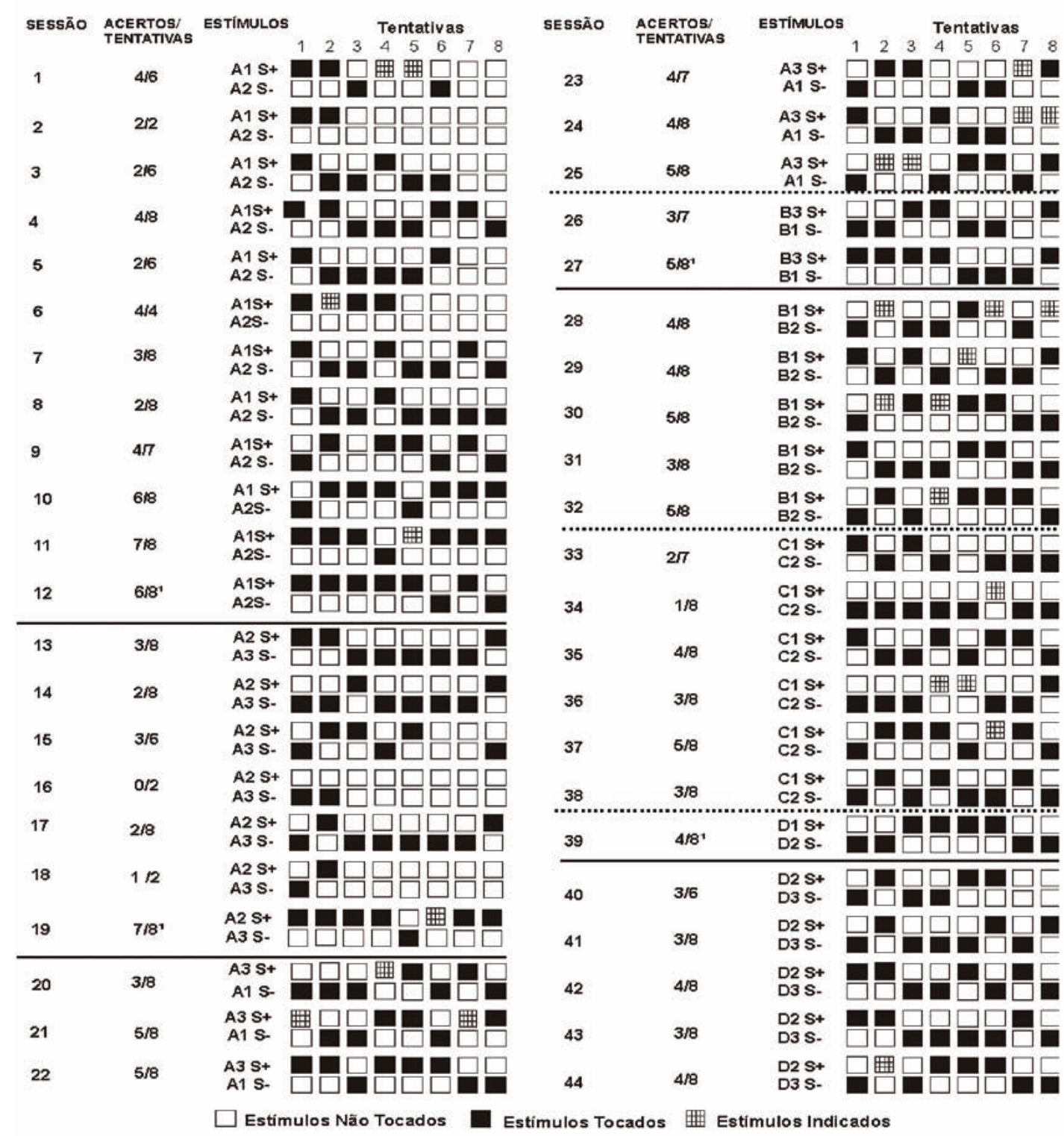

Figura 3. Respostas por tentativas nas 44 sessões de P10. O item acertos/tentativas corresponde aos acertos pelo número de tentativas completadas na sessão. As linhas horizontais contínuas indicam a mudança na função dos estímulos e as pontilhadas, mudança no grupo de estímulos treinados. O critério de aprendizagem de 4 rcc é indicado pelo número 1 sobrescrito no item acerto/ tentativa. 'Estímulos Tocados’ caracterizam as respostas sem prompts e ‘Estímulos Indicados’, as respostas após prompts.

alcançado. Como nas três primeiras sessões P10 apresentou poucos acertos e não completou todas as oito tentativas por sessão, também no seu treino foi providenciada uma redução da estimulação no ambiente experimental (a partir da sessão 4). Essa redução inicial parece ter favorecido o engajamento de P10 na tarefa e seu desempenho, como pode ser visto pelo aumento de sessões completadas e de respostas aos $\mathrm{S}+(\mathrm{s})$ (ver sessões 4 a 12).

A partir da sessão 21 em diante, observou-se que P10 começou a mostrar desinteresse pela tarefa (necessitando de prompts para terminar as tentativas, respondendo aos estímulos sem indícios de resposta de observação cuidadosa, concluindo mais rapidamente as sessões e buscando o cuidador no sofá). Conforme mencionado antes, essa situação foi inicialmente contornada pela substituição dos estímulos do conjunto A pelos do conjunto B. Além disso, procurando manter o bom desempenho obtido com a substituição do conjunto de estímulos, a partir da sessão 27 um conjunto de medidas foram tomadas. Primeiro, os vídeos passaram a ser trocados a cada duas sessões e a ter uma duração de cerca de $10 \mathrm{~s}$, sendo apresentadas sequências de filmes na sessão. Em algumas sessões (29, 30, 32, 33 e 36) foi solicitado que a mãe de P10 participasse do experimento, com as mesmas funções que a experimentadora (em função da aparente perda do valor reforçador das interações com a experimentadora). A partir da sessão 30 , os poucos brinquedos que restavam na sala foram retirados do campo visual de P10 durantes as sessões. Por último, procurando ampliar o rol de estímulos reforçadores, a partir da sessão 39, após as respostas corretas, 
Tabela 4. Número de tentativas e de sessões nas etapas do treino de DSMS de P12.

\begin{tabular}{ccccc}
\hline \multicolumn{3}{c}{ Estímulos } & & \\
\cline { 1 - 2 } S+ & S- & S- & Tentativas & Sessões \\
\hline A1 & A2 & - & 117 & $15^{*}$ \\
B1 & B2 & - & 8 & 1 \\
B2 & B3 & - & 7 & 1 \\
B3 & B1 & - & 37 & 5 \\
B1 & B2 & B3 & 38 & $5^{*}$ \\
C1 & C2 & C3 & 08 & $1 *$ \\
& TOTAL & & $\mathbf{2 1 5}$ & $\mathbf{2 8}$ \\
\hline
\end{tabular}

* Não foi alcançado nenhum dos critérios de aprendizagem

P10 passou a ser convidado a brincar com alguns brinquedos (retirados de uma caixa) com a experimentadora.

Com a exceção de uma nova substituição dos conjuntos de estímulos (do conjunto $\mathrm{C}$ pelo $\mathrm{D}$, como mencionado antes) na sessão 39, todas as medidas tomadas após a sessão 27 não afetaram de forma significativa o desempenho de P10. Devese salientar, ainda, que enquanto P14 e P12 (ver Figuras 2 e 4) tiveram apenas, respectivamente, seis e três sessões com prompts, $\mathrm{P} 10$ teve 17 sessões nas quais foram necessárias dicas físicas e/ou visual para que a resposta ocorresse. No entanto, os efeitos desses prompts sobre o desempenho de P10 não foi claro, gerando respostas corretas em metade das vezes em que foram usados e respostas erradas na outra metade.

Como mostra a Tabela 4, P12 passou pelo treino de quatro arranjos de DS com dois estímulos, alcançando um dos critérios de aprendizagem em três arranjos (B1/B2, B2/B3, B3/B1), sendo que dois implicavam mudança na função de estímulo. O participante P12 foi exposto ainda a dois arranjos de discriminação simples com três estímulos sem alcançar nenhum dos critérios de aprendizagem. Foram usados três conjuntos de estímulos, com as substituições sendo feitas em função da dificuldade em alcançar um dos critérios de aprendizagem com certo conjunto.

A Figura 4 demonstra as respostas em todas as tentativas das 28 sessões realizadas com P12. Esse participante iniciou o experimento depois dos outros dois, e seu treino já começou com a redução da estimulação no ambiente experimental. Apesar disso, ele apresentou dificuldades na tarefa com o conjunto A de estímulos, realizando 15 sessões sem êxito com a primeira DS treinada (A1/A2). Após essa extensa exposição ao arranjo A1/A2, foi realizada a substituição desses estímulos pelos estímulos do conjunto B (definindo-se que seriam feitas substituições dos conjuntos de estímulos a cada cinco sessões realizadas sem que fosse alcançado um dos critérios de aprendizagem). Na $1^{\mathrm{a}}$ e $3^{\mathrm{a}}$ tentativas da sessão 19 , na $3^{\text {a }}$ tentativa da sessão 25 , na $3^{\mathrm{a}}$ e $4^{\mathrm{a}}$ tentativas da sessão 26 e na $3^{\text {a }}$ tentativa da sessão 27 , P12 escolheu simultaneamente o estímulo S+ e um S-, o que causou a anulação dessas tentativas. Logo na primeira sessão (16) em que houve a substituição do conjunto (para B1/B2), P12 alcançou um dos critérios de aprendizagem, passando para o treino da primeira mudança (B2/B3). Essa discriminação, com mudança na função de estímulo, também foi realizada com sucesso em apenas uma sessão (17).

A partir da sessão 17, os vídeos utilizados como consequência para resposta corretas foram apresentados em sequência e tiveram a duração de aproximadamente 10 s, sendo substituídos, no máximo, a cada três sessões. Essa alteração, somada à substituição dos conjuntos de estímulo, parece ter favorecido o bom desempenho no treino de DSMS com dois estímulos (ver sessões 16 a 22). No entanto, como no caso de P14, nem mesmo a junção dessas alterações (somadas ainda à redução de estimulação no ambiente experimental) favoreceu o desempenho na tarefa de discriminação com três estímulos (ver sessões 23 a 28). O participante P12 também recebeu feedbacks para escolhas incorretas a partir da sessão 19 (os erros foram consequenciados com verbalizações corretivas do tipo "não foi o desenho certo"). Mas, de forma semelhante ao observado com P14, esse procedimento não pareceu efetivo para favorecer a discriminação das respostas corretas.

Os resultados dos três participantes, após aproximadamente quatro meses de treino, podem ser assim sintetizados: P14 passou pelo treino de oito arranjos de DS com dois estímulos, alcançando um critério de aprendizagem em seis deles. Desses seis, três implicavam mudança na função de estímulos; P10 passou pelo treino de oito arranjos de DS com dois estímulos, alcançando um critério de aprendizagem em quatro (um apenas implicando mudança na função de estímulo); P12 passou pelo treino de quatro arranjos de DS com dois estímulos, alcançando um dos critérios de aprendizagem em três arranjos (dois implicando mudança na função de estímulo). Os participantes P14 e P12 passaram ainda pelo treino de dois arranjos de DS com três estímulos sem alcançar nenhum dos critérios de aprendizagem.

\section{Discussão}

O presente estudo procurou analisar, em bebês entre os 10 e 20 meses de vida, a aprendizagem de discriminações simples com mudanças sucessivas na função dos estímulos. A aprendizagem desse repertório pode facilitar o ensino posterior de discriminações condicionais por identidade que, por sua vez, podem funcionar como base para o desenvolvimento de repertórios simbólicos mais complexos (tais como identidade generalizada, discriminação arbitrária e equivalência de estímulos) (Barros \& cols., 2003, Boelens \& cols., 2000; Zygmont \& cols., 1992). Analisou-se, ainda, o efeito de algumas variáveis (e.g., redução de estímulos no ambiente, variação de estímulos reforçadores) na manutenção dos participantes no ambiente experimental e na aprendizagem da tarefa.

Os resultados mostraram que os participantes somente alcançaram a etapa inicial do treino de DSMS planejado: P14 e P12 mostraram evidência da aprendizagem de DSMS com dois estímulos e P10, da aprendizagem de DS com dois estímulos. Os participantes $\mathrm{P} 14$ e P12 chegaram à etapa de treino de DS com três estímulos, mas não alcançaram nenhum dos critérios de aprendizagem. Esses resultados confirmam 


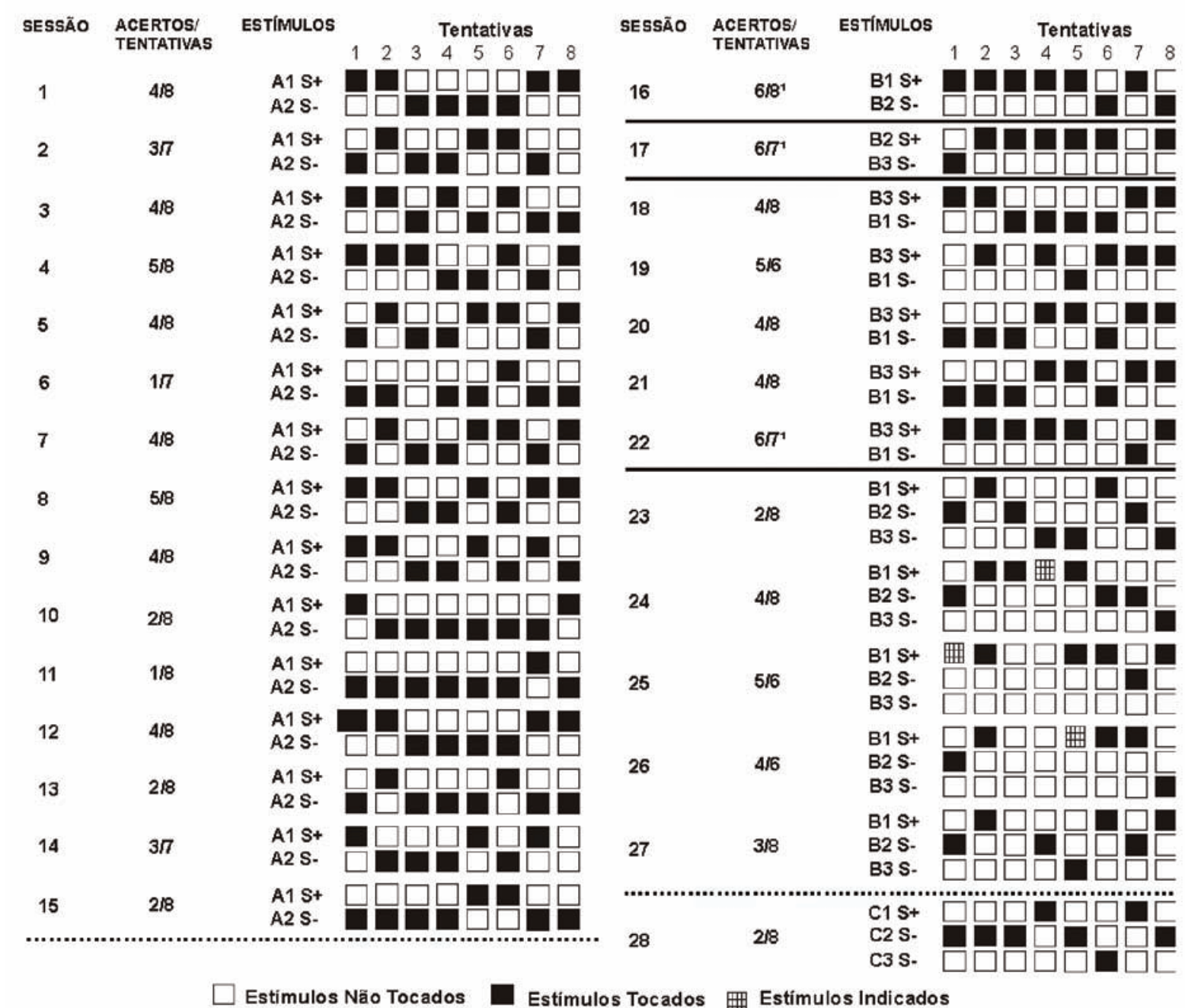

Figura 4. Respostas por tentativas nas 28 sessões de P12. O item acertos/tentativas corresponde aos acertos pelo número de tentativas completadas na sessão. As linhas horizontais contínuas indicam a mudança na função dos estímulos e as pontilhadas, mudança no grupo de estímulos treinados. O critério de aprendizagem de 4 rcc é indicado pelo número 1 sobrescrito no item acerto/tentativa. 'Estímulos Tocados' caracterizam as respostas sem prompts e ‘Estímulos Indicados', as respostas após prompts.

a possibilidade de ensinar DS para crianças com menos de dois anos de idade (Gil \& cols., 2006; Oliveira \& Gil, 2008) e demonstram que o repertório de DSMS também pode ser ensinado para crianças nessa etapa do desenvolvimento (i.e., no período inicial de aquisição da linguagem).

Diversos procedimentos apontados por Gil e colaboradores (e.g., Gil \& cols., 2006; Oliveira \& Gil, 2008) como facilitadores da manutenção das crianças na situação experimental e, consequentemente, da aprendizagem da tarefa, foram empregados no presente estudo: a redução da estimulação no ambiente experimental; a realização de um número pequeno de tentativas por sessão (8); estabelecimento de critérios flexíveis de aprendizagem; a presença de um adulto familiar durante as sessões; e interações com o experimentador (em função de respostas corretas) entre tentativas.

Além dos procedimentos indicados por Gil e colaboradores, foram aplicados outros procedimentos também procurando favorecer a manutenção das crianças na situação experimental e a aprendizagem da tarefa: a substituição dos conjuntos de estímulos usados nos treinos; uso de feedbacks (correções) e prompts quando ocorriam respostas erradas; e a substituição de parte das consequências para acerto (os filmes/desenhos) a cada duas ou três sessões.

A redução da estimulação no ambiente experimental esteve relacionada com melhorias no desempenho dos participantes nas sessões seguintes à sua realização (com exceção de P12, que teve problemas com os estímulos do conjunto A). Da mesma forma, a realização de poucas tentativas por sessão, a substituição de parte das consequências para acertos a cada duas ou três sessões e o estabelecimento de critérios flexíveis de aprendizagem, ao tornarem menos complexas as contingências de aprendizagem, também parecem ter favorecido um bom desempenho das crianças na etapa inicial do treino. Por outro lado, o uso de feedbacks (correções) e prompts para respostas erradas e a participação do cuidador na sessão experimental não pareceram afetar o desempenho dos participantes.

Entre os procedimentos utilizados, aquele que pareceu ser mais efetivo para garantir um bom desempenho da criança foi a substituição dos conjuntos de estímulos discriminativos. Por 
exemplo, na sessão 39 de P10 e na sessão 16 de P12 houve a aprendizagem da discriminação justo com a substituição do conjunto de estímulos, e na sessão 27 de P10, logo após a substituição. No entanto, da mesma forma que os demais procedimentos utilizados neste estudo, a substituição dos conjuntos de estímulos não foi suficiente para garantir a aprendizagem da DS com três estímulos.

Conforme observado antes, os resultados de P14 e P12 mostraram uma queda no desempenho quando foi introduzido o treino de DS com três estímulos. Um resultado similar tem sido observado com macacos-prego (Dahás, 2007; Fonseca, 2007; Rico, 2006). Uma possível explicação para essa dificuldade pode estar no tipo de controle de estímulo que os arranjos de DS com dois e três estímulos permitem. No treino com dois estímulos, as respostas dos participantes podem ficar sob o controle discriminativo apenas dos estímulos positivos (S+), caracterizando o controle por 'seleção'; sob o controle discriminativo apenas dos estímulos negativos (S-), caracterizando o controle por 'rejeição’; ou ainda sob o controle de ambos os estímulos, caracterizando um controle misto (seleção dos S+ e rejeição dos S-). Goulart, Mendonça, Barros, Galvão e McIlvane (2005) observaram que tanto o controle por seleção como por rejeição pode surgir no treino de DS com macacos-prego.

Em um treino de DS com dois estímulos, ambos os tipos de controle podem ser efetivos para gerar um bom desempenho. No entanto, em um treino de DS com mais de dois estímulos, o controle por rejeição implica em um custo de resposta maior, que pode prejudicar o desempenho nessa tarefa. Além disso, em treinos nos quais os estímulos podem mudar de função a cada sessão ou tentativa (DSMS ou discriminações condicionais), o estabelecimento de um único tipo de controle pode gerar desempenhos corretos, mas que não são coerentes com as relações de controle de estímulo estabelecidas pelos experimentadores (McIlvane \& Dube, 2003).

De acordo com McIlvane e Dube (2003), análises das relações de controle de estímulo estabelecidas em treinos discriminativos (simples ou condicionais) permitem aumentar a probabilidade de haver coerência entre as relações de controle de estímulo planejadas e aquelas que efetivamente ganham controle sobre o comportamento dos indivíduos. Assim, pode ser possível minimizar a dificuldade encontrada no repertório de discriminação com três estímulos por meio da observação criteriosa dos erros em função das relações de controle vigentes durante os treinos com dois estímulos. A partir disso, pode-se procurar modificar o controle de estímulos, buscando torná-lo coerente com o planejado.

Deve-se ressaltar, no entanto, que com o desenvolvimento do treino (que teve uma duração de quase quatro meses), os três participantes começaram a demonstrar menos interesse pela tarefa e os filmes/desenhos e as interações verbais com a experimentadora mostraram-se menos efetivos para manter a criança no ambiente experimental tanto quanto para fortalecer respostas corretas. A diminuição do tempo gasto na tarefa (com o consequente aumento do tempo gasto em atividades concorrentes) e a aparente diminuição no efeito reforçador dos estímulos empregados no presente estudo indicam a necessidade do estabelecimento de treinos mais breves e que enfatizem mais o repertório que está sendo ensinado (e menos os estímulos). Isso implica buscar procedimentos alternativos para se chegar ao ensino de discriminações condicionais.

Em um estudo longitudinal que objetivou identificar, em situação natural, as variáveis apontadas como pré-requisitos para aquisição de repertório simbólico, Souza e Pontes (2007) verificaram uma elevada frequência de nomeações dos objetos/eventos na interação criança-acompanhante. Foi observado que as crianças, com 2 meses de vida no início do estudo, foram expostas diariamente a vários exemplares de relações nome-referente; com o passar dos meses, o número de exemplares desse tipo de relação apresentados às crianças aumentou consideravelmente. A análise desses resultados sugere que, em situações naturais, o processo de aquisição de repertórios pré-simbólicos e simbólicos parece depender mais de uma exposição a múltiplos exemplares de certo tipo de relação de controle de estímulos que caracteriza determinados repertórios (e.g., discriminação condicional por identidade, arbitrária) do que da aprendizagem de relações de estímulos específicas. Essa ênfase no tipo de repertório ensinado pode ser uma melhor estratégia para se obter um responder generalizado às diferentes relações de controle de estímulos constitutivas de repertórios pré-simbólicos e simbólicos. Os bons resultados obtidos com a substituição dos conjuntos dos estímulos no presente estudo parecem favorecer essa suposição.

Estudos recentes (Berens \& Hayes, 2007; Luciano, Becerra \& Valverde, 2007) têm mostrado evidências de que a exposição a múltiplos exemplares de determinadas relações de estímulos pode favorecer a aprendizagem do responder generalizado para esses tipos de relações. Berens e Hayes, ao utilizarem um procedimento que combinou o treino de múltiplos exemplares (TME) e testes de generalização empregando estímulos novos e já treinados, verificaram a aprendizagem de relações arbitrárias e a generalização desse repertório em crianças de 4 a 5 anos de idade.

Luciano e cols. (2007) avaliaram o efeito do TME em simetria receptiva (ver um objeto e escutar o seu nome, e depois escutar o nome do objeto e selecioná-lo) sobre a aquisição de simetria receptiva generalizada e sobre a emergência de relações de equivalência entre estímulos visuais, em uma criança com 15 meses e 24 dias no início do estudo. O Experimento 1 verificou que após uma história de TME em simetria receptiva com 10 estímulos, a criança apresentou simetria receptiva com cinco dos seis novos objetos utilizados no teste. Os experimentos 2 e 3 constataram a emergência de relações de equivalência entre estímulos visuais com dois (aos 19 meses) e três conjuntos de dois estímulos cada, respectivamente.

Um experimento (Comesanha \& Souza, 2008) da linha de pesquisa na qual se insere o presente estudo mostrou resultados promissores ao utilizar um procedimento de TME para estabelecer repertórios de discriminação condicional por identidade (DCI) em três crianças com até 20 meses de idade. Em uma situação experimental semelhante à do estudo atual (estímulos GIFs apresentados em uma tela sensível ao toque) eram realizadas três tentativas por dia. Inicialmente era apresentado o mesmo estímulo S+ em duas tentativas seguidas (os estímulos S- eram trocados a cada tentativa). Após o alcance de um dos critérios de aprendizagem (três respostas corretas consecutivas ou cinco corretas em seis 
respostas), a cada tentativa eram apresentados novos $\mathrm{S}+\mathrm{e}$ S-. Essas características do procedimento, junto com outras usadas no presente estudo (e.g., redução de estímulos no ambiente durante a tarefa experimental, interações com o experimentador após cada tentativa e ao final da tarefa e uso de uma grande diversidade de filmes/desenhos animados como reforços) parecem ter contribuído para manter as crianças interessadas na tarefa experimental e para que duas delas alcançassem os critérios de aprendizagem seguidas vezes no treino com novos S+ e S-.

O andamento dessa linha de pesquisa procurará avaliar se o tipo de procedimento utilizado por Comesanha e Souza (2008) é adequado para estabelecer repertórios de discriminação condicional por identidade generalizada e discriminação condicional arbitrária em crianças com menos de dois anos de idade.

\section{Referências}

Augustson, K. G., \& Dougher, M. J. (1991). Teaching conditional discrimination to young children: Some methodological success and failures. Experimental Analysis of Human Behavior Bulletin, 9, 21-24.

Barros, R. S., Galvão, O. F., \& McIlvane, W. J. (2003). The search for relational learning capacity in Cebus apella: A programmed “educational” approach. Em Sal Soraci Jr. \& Kimiyo Murata-Soraci (Eds.), Visual information processing (pp. 223-245). Westport: Praeger Publishers.

Bates, E., \& Elman, J. L. (2000). The ontogeny and phylogeny of language: A neural network perspective. Em S. Parker, J. Langer \& M. McKinney (Eds.), Biology, brains, and behavior: The evolution of human development (pp. 89-130). Santa Fe: School of American Research Press.

Berens, N. M., \& Hayes, S. C. (2007). Arbitrarily applicable comparative relations: Experimental evidence for a relational operant. Journal of Applied Behavior Analysis, 40, 45-71.

Boelens, H., Broek M. V. D., \& Klarenbosch, T. V. (2000). Symmetric matching to sample in 2-year-old children. The Psychological Record, 50, 293-304.

Bremner, G, \& Fogel, A. (Orgs.) (2004). Blackwell handbook of infant development. London: Blackwell Publishing.

Carr, D., Wilkinson, K. M., Blackman, D., \& McIlvane, W. J. (2000). Equivalence classes in individuals with minimal verbal repertoires. Journal of the Experimental Analysis of Behavior, 74, 101-114.

Comesanha, O., \& Souza. C. B. A. (2008). Pareamento ao modelo por identidade em bebês [CD-ROM] Em Resumos da XXXVIII Reunião Anual de Psicologia. Uberlândia: Universidade Federal de Uberlândia.

Dahás, L. (2007). Discriminação simples e pareamento ao modelo com estímulos bidimensionais em Cebus apella infante. Trabalho de Conclusão de Curso, Universidade Federal do Pará, Belém.

Deacon, T.W. (1997). The symbolic species: The co-evolution of language and the brain. New York: Norton.

Devany, J. M., Hayes, S. C., \& Nelson, R. O. (1986). Equivalence class formation in language-able and language-disable children. Journal of the Experimental Analysis of Behavior, 46, 243-257.
Fonseca, A. R. (2007). Discriminação simples e pareamento ao modelo com objetos em Cebus apella. Trabalho de Conclusão de Curso, Universidade Federal do Pará, Belém.

Gil, M. S. C. A., \& Oliveira, T. P. (2003). Um procedimento de treino de discriminação condicional com bebês. Em M. Z. S. Brandão, F. C. S. Conte, F. S. Brandão, Y. K. Ingberman, C. B. Moura, V. M. Silva \& S. M. Oliane (Orgs.), Sobre comportamento e cognição. Clínica, pesquisa e aplicação - Vol. 12 (pp. 469-477). Santo André: ESETec.

Gil, M. S. C. A., Oliveira, T. P., Sousa, N. M., \& Faleiros, D. A. M. (2006). Variáveis no ensino de discriminação para bebês. Psicologia: Teoria e Pesquisa, 22, 143-152.

Goulart, P. R. K., Mendonça, M. B., Barros, R. S., Galvão, O. F., \& McIlvane, W. J. (2005). A note on select - and reject - controlling relations in the simple discrimination of capuchin monkeys (Cebus Apella). Behavioural Process, 69, 295-302.

Hauser, M. D., Chomsky, N., \& Fitch, W. T. (2002). The faculty of language: What is it, who has it, and how did it evolve? Science, 298, 1569-1579.

Hayes, S. C., Barnes-Holmes, D., \& Roche, B. (2001). Relational frame theory: A post-skinnerian account of human language and cognition. New York: Kluwer Academic/Plenum.

Horne, P. J., \& Lowe, C. F. (1996). On the origens of naming and other symbolic behavior. Journal of the Experimental Analysis of Behavior, 65, 185-241.

Lipkens, R., Hayes, S. C., \& Hayes, L. J. (1993). Longitudinal study of the development of derived relations. Journal of Experimental Child Psychology, 56, 201-239.

Luciano, C., Becerra, I. G., \& Valverde, M. R. (2007). The role of multiple-exemplar training and naming in establishing derived equivalence in an infant. Journal of the Experimental Analysis of Behavior, 87, 349-365.

McIlvane, W. J., \& Dube, W. V. (2003). Stimulus control topography coherence theory: Foundations and extensions. The Behavior Analyst, 26, 195-213.

O’Donnell, J. O., \& Saunders, K. J. (2003). Equivalence relations in individuals with language limitations and mental retardation. Journal of the Experimental Analysis of Behavior, 80, 131-157.

Oliveira, T. P., \& Gil, M. S. C. A. (2008). Condições experimentais facilitadoras para a aprendizagem de discriminação por bebês. Psicologia: Teoria e Pesquisa, 24, 5-18.

Rico, V. V. (2006). Persistência comportamental e topografía de controle de estímulos coerente em treino de discriminação simples e escolha condicional por identidade ao modelo com quatro escolhas em macaco-prego (Cebus apella). Dissertação de Mestrado, Universidade Federal do Pará, Belém.

Rovee-Collie, C., \& Barr, R. (2004). Infant learning and memory. Em G. Bremner \& A. Fogel (Orgs.), Handbook of infant development (pp. 139-168). London: Blackwell Publishing.

Savage-Rumbaugh, E., Murphy, J., Sevcik, R., Brakke, K., Williams, S., \& Rumbaugh, D. (1993). Language comprehension in ape and child. Monographs of the Society for Research in Child Development, 58, 1-256.

Sidman, M. (2000). Equivalence relations and reinforcement contingency. Journal of the Experimental Analysis of Behavior, 74, 127-146.

Sidman, M., \& Tailby, W. (1982). Conditional discrimination vs. matching to sample: An expansion of the testing paradigm. Journal of the Experimental Analysis of Behavior, 37, 5-22. 
Sidman, M., Wynne, C. K., Maguire, R. W., \& Barnes, T. (1989). Functional classes and equivalence relations. Journal of the Experimental Analysis of Behavior, 52, 261-274.

Slater, A. (2004). Visual perception. Em G. Bremner \& A. Fogel (Orgs.), Handbook of infant development (pp. 5-34). London: Blackwell Publishing.

Souza, C. B. A., Borges, R. P., Goulart, P. R. K., Barros, R. S., \& Galvão, O. F., (2009). Testes de identidade generalizada com objetos em macaco-prego (Cebus apella). Psicologia: Teoria e Pesquisa, 25, 169-178.

Souza, C. B. A., \& Pontes, S. S. (2007). Variações paramétricas em pré-requisitos da linguagem: Estudo longitudinal das interações criança-acompanhante. Interação em Psicologia, 11, 55-70.

Tomasello, M., \& Call, J. (1997). Primate cognition. Nova Iorque: Oxford University Press.

Wilkinson, K. M., \& Green, G. (1998). Implications of "fast mapping” for vocabulary expansion in individuals with mental retardation. Augmentative and Alternative Communication , 14 , $162-170$.
Zentall, T. R., Galizio, M., \& Critchfield, T. S. (2002). Categorization, concept learning, and behavior analysis: An introduction. Journal of the Experimental Analysis of Behavior, 78, 237-248.

Zygmont, D. M., Lazar, R. M., Dube, W. V., \& McIlvane, W. J. (1992). Teaching arbitrary matching via sample stimulus-control shaping to young children and mentally retarded individuals: A methodological note. Journal of the Experimental Analysis of Behavior, 57, 109-117.
Recebido em 21.05.08

Primeira decisão editorial em 11.03.09

Versão final em 30.03.09

Aceito em 11.05 .09 